Published online 2015 April 21.

Case Report

\title{
Chromosomally and Anatomically Normal Fetuses With Increased First Trimester Nuchal Translucency Conceived by ICSI
}

\author{
Maryam Niknejadi ${ }^{1,} ;$ Hadieh Haghighi $^{1}$ \\ ${ }^{1}$ Department of Reproductive Imaging, Reproductive Biomedicine Research Center, Royan Institute for Reproductive Biomedicine (ACECR), Tehran, Iran \\ ${ }^{*}$ Corresponding author: Maryam Niknejadi, Department of Reproductive Imaging, Reproductive Biomedicine Research Center, Royan Institute for Reproductive Biomedicine \\ (ACECR), Tehran, Iran P. O. Box: 19395-4644, Tehran, Iran. Tel:+98-212356246, E-mail: m.niknejadi@royaninstitute.org \\ Received: July 8, 2012; Revised: October 24, 2012; Accepted: December 9, 2012
}

\begin{abstract}
Nuchal translucency (NT) measurements in the first trimester screening between 11 and 14 weeks' gestation are regarded as a clear marker for aneuploidies. The presence of a thickened NT, even if the karyotype is normal, can be associated with structural abnormalities. Having an abnormal screening of NT, parents and physicians could face dilemma over abortion particularly in a case of IVF/ICSI fetuses. Measurement of the NT thickness combined with biochemical markers has a false-positive rate of 5\%. Hereby we present six cases of chromosomally normal fetuses with an increased NT thickness in the first trimester, a normal karyotype and normal follow-up scans, who had a good prognosis for a normal early childhood. This report may help increase the confidence of couples who are reluctant to terminate the pregnancy.
\end{abstract}

Keywords: Congenital Abnormalities; Prenatal Diagnosis; Nuchal Translucency Measurement

\section{Introduction}

Fetal nuchal translucency (NT) refers to the sonographic appearance of subcutaneous edema in the fetal neck measured according to the fetal medicine foundation (FMF) guidelines. NT is defined as the maximal thickness of the sonolucent zone (fluid accumulation) between the inner aspect of the fetal skin and the outer aspect of the soft tissue overlying the cervical spine or the occipital bone (Figure 1). To avoid false negative or positive results, the fetus should be in a neutral position, with the head in line with the spine. During the scan, more than one measurement must be taken and the maximum one that meets the criteria should be considered.

For this report, the outcome of 703 pregnancies presenting at Royan Institute after intra cytoplasmic sperm injection (ICSI) between the years 2008 and 2009 were reviewed. First trimester measurements of NT were performed during the routine first trimester screening for 856 fetuses. A total of 12 fetuses with an increased NT(NT $\geq$ 3) were considered for further investigation. In the followup ultrasounds, six out of 12 fetuses showed no anomalies and the result of amniocentesis (karyotype), fetal echocardiography and triple test or double test (the biochemical markers of pregnancy-associated plasma protein-A and free $\beta$-human chorionic gonadotropin) were also normal. Six healthy neonates were followed during their first and second year by routine pediatrician visits and the mental, physical or motor developmental delay was not observed in any of the cases and their functions were normal.

All sonographic measurements were carried out by an expert radiologist with 10 years of experience and FMF certification in the measurement of NT. Images were saved and re-checked by another expert radiologist. Increased NT can be associated with a high incidence rate of chromosomal and non-chromosomal abnormalities. However, in nine studies that have addressed the issue of pediatric long-term follow-up of chromosomally and anatomically normal fetuses with increased NT, this measurement was suggested to be an indefinite indicator for the evaluation of fetal anomalies (1-9).

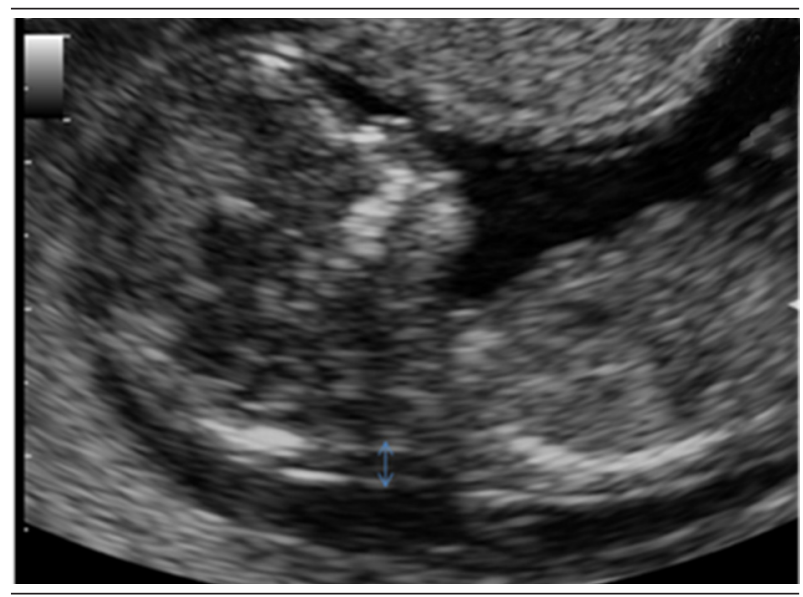

Figure 1. According to the FMF guidelines, NT is measured as the maximal thickness of the sonolucent zone (fluid accumulation) between the inner aspect of the fetal skin and the outer aspect of the soft tissue overlying the cervical spine or the occipital bone.

Copyright ( ) 2015, Tehran University of Medical Sciences and Iranian Society of Radiology. This is an open-access article distributed under the terms of the Creative Commons Attribution-NonCommercial 4.0 International License (http://creativecommons.org/licenses/by-nc/4.0/) which permits copy and redistribute the material just in noncommercial usages, provided the original work is properly cited. 


\section{Case Presentation}

\subsection{Case 1}

A 28-year-old woman with a twin pregnancy, which was conceived by ICSI, was referred for routine screening test in the first trimester. The result of semen analysis showed a low motility (35\%) with a high abnormal morphology (87\%). In the routine first trimester screening at 12 weeks and 2 days of gestation, the NT measurements of the first fetus was $3.4 \mathrm{~mm}$ (normal range for this age is 1.2$3.1 \mathrm{~mm}$ ). Although she refused to undergo the triple test and we had no result for amniocentesis (karyotype) and fetus echocardiography, follow-up ultrasounds revealed normal results. Two healthy babies (boy and girl) were born at 37 weeks of pregnancy. The weights of the newborns were 2400 grams and 3400 grams. Neonates were followed during the first 2 years of their life and none of them had developmental delay.

\subsection{Case2}

A 35-year-old woman, who was conceived by ICSI, was referred for the routine screening test during her pregnancy. Her obstetric and medical history revealed primary infertility for a period of 7 years, mild endometriosis and polycystic ovaries. The result of semen analysis indicated low motility (35\%) and high abnormal morphology (88\%). During the routine first trimester screening at 13 weeks of gestation, NT was measured at $3 \mathrm{~mm}$. The normal range of NT for this age is 1.6-2.4 mm. Nuchal skin fold (NF) measurements and prenatal follow-up ultrasound findings were normal. A Triple test was performed, and it showed a positive result and a high risk of trisomy 21 . The patient was referred for amniocentesis or chorionic villus sampling. The amniocentesis findings were normal and a healthy baby girl (weight $=3600$ grams) was born after a full-term preg nancy. She was followed during the first and second year of life and no developmental delay was detected.

\subsection{Case 3}

A 33-year-old pregnant woman conceived by ICSI was referred for a routine screening test. Her obstetric and medical histories were unremarkable. The result of semen analysis indicated low motility (30\%) and high abnormal morphology (81\%). The result of the routine first trimester screening at 11 weeks and 5 days gestation showed a significant increased NT $(\mathrm{NT}=5)$. The normal range of NT for this age is $1-2.8 \mathrm{~mm}$. Later examination revealed that collection of fluid was not confined to the neck but was enveloped throughout the fetus. The result of follow-up such as prenatal ultrasound findings, result of an amniocentesis (karyotype) and echocardiography were reported normal. She gave birth to a healthy baby boy (weight $=3500$ grams) at 37 weeks of pregnancy by elective cesarean section at term. Follow-up after birth till 2 years has revealed no developmental delay.

\subsection{Case 4}

A 26-year-old woman who was conceived by ICSI was referred for a routine screening test during her pregnancy. Her obstetric and medical history showed 8 years of primary infertility and polycystic ovaries. The result of semen analysis showed a low sperm concentration with a low total sperm count, low motility (30\%) and high abnormal morphology (86\%). First trimester measurement of NT at 12 weeks of gestation was $3.2 \mathrm{~mm}$ during the routine first trimester screening. The normal range of NT for this age is 1.1-3 mm. Although she refused to undergo amniocentesis (karyotype) and fetal echocardiography, triple test and follow-up prenatal ultrasound findings were normal. She gave birth at term to a healthy baby boy at 38 weeks (weight $=3500$ grams). The 2-year follow-up after birth revealed no developmental delay.

\subsection{Case 5}

Following the routine screening test during pregnancy, a 31-year-old woman (conceived by ICSI) was referred to this institute. Her obstetric and medical history apart from 7 years primary infertility was unremarkable. The result of semen analysis revealed a low motility (35\%) and a high abnormal morphology (92\%). The result of routine first trimester screening at 12 weeks and 5 days of gestation showed an increased NT (3.3 mm) and generalized edema. The normal range of NT for this age is $1.5-3 \mathrm{~mm}$. Although she refused to do the triple test and fetal echocardiography, follow-up prenatal ultrasound findings and amniocentesis (karyotype) were normal. She gave birth to a healthy term baby girl (weight $=3400$ grams). Follow-up after birth revealed no developmental delay.

\subsection{Case 6}

A 30-year-old patient (conceived by ICSI) first presented to our institute at 12 weeks and 1 day of gestation and a twin pregnancy was detected. The second fetus was diagnosed with an increased NT (NT $=3.5 \mathrm{~mm})$. The normal range of NT for this age is $1.2-3 \mathrm{~mm}$. The couples' history was significant for abnormal semen analysis. The result of semen analysis showed a sperm motility of $35 \%$ and an abnormal morphology of $80 \%$. In this case, follow-up prenatal ultrasound findings and triple test were normal. Amniocentesis (karyotype) and echocardiography were cancelled because the patient refused to do so. Two healthy babies (boy and girl) were born at 37 weeks of pregnancy. The weights of the newborns were 2400 grams and 2900 grams. The neonates were followed for the first and second year of their life and none of them had developmental delay.

\section{Discussion}

Although measurement of the NT thickness combined with biochemical markers has a false-positive rate of 5\% (10), it is regarded as a basic screening test with high sen- 
sitivity for identifying fetuses at risk for aneuploidy $(1,11$, 12). However, NT is increased in $4.4 \%$ of euploid fetuses who are at risk for fetal anomalies and an adverse pregnancy outcome (12).

For this report first trimester ultrasound screening was performed for 703 women with a total number of 856 fetuses who were conceived by ICSI. First trimester measurement of NT was performed for 856 fetuses and 12 out of 856 fetuses with increased NT (NT $\geq 3$ ) were taken for further investigation. In this report, six out of 12 cases who were chromosomally and anatomically normal fetuses with increased first trimester NT were described in detail. All these six fetuses had favorable prognosis despite the increased NT. We had no chromosomal report of the remained six fetuses because of reduction due to multiple pregnancies (4/12) and intrauterine fetal death (IUFD 2/12). The chance of delivering a healthy baby decreases with NT thickness from approximately 70\% for an NT of 3.5-4.4 $\mathrm{mm}$ to about $15 \%$ for an NT of $6.5 \mathrm{~mm}$ or more (13). Based on previous studies, fetuses with increased NT (less than $4 \mathrm{~mm}$ ), normal karyotype and normal findings in the 20-week scan, showed a markedly increased favorable outcome (14). After a normal detailed genetic sonography and fetal echocardiography, the incidence of adverse outcome and developmental delay were not significantly different from the normal population (9). According to studies performed by Souka et al. fetuses with increased NT thickness have a high risk of adverse or poor pregnancy outcome $(1,13)$. In this study, we only had one case of increased NT and intrauterine fetal death (IUFD) and one case of premature rupture of the membranes (PROM).

This report is limited in number and should be further completed. In this series, semen analysis of all six cases were abnormal with a low motility of $30-35 \%$ and a high abnormal morphology of $80-92 \%$. Although specific semen preparation technique in the case of impaired semen parameters in ART cycles is advisable, couples diagnosed with male-factor infertility should receive genetic counseling about the increased risk of congenital abnormalities before IVF-ICSI (15). This report provided six cases of increased NT with normal outcomes. It may help increase the confidence of couples who are reluctant to terminate the pregnancy. However, caution is necessary in cases of increased NT and parents should be offered a detailed fetal evaluation (genetic sonography) at the end of the first trimester and also at 18-22 weeks of gestation. Triple test, amniocentesis, fetal echocardiography and follow-up after birth are necessary.

\section{References}

1. Souka AP, Krampl E, Bakalis S, Heath V, Nicolaides KH. Outcome of pregnancy in chromosomally normal fetuses with increased nuchal translucency in the first trimester. Ultrasound Obstet Gynecol. 2001;18(1):9-17.

2. Van Vugt JM, Tinnemans BW, Van Zalen-Sprock RM. Outcome and early childhood follow-up of chromosomally normal fetuses with increased nuchal translucency at 10-14 weeks' gestation. Ultrasound Obstet Gynecol.1998;11(6):407-9.

3. Brady AF, Pandya PP, Yuksel B, Greenough A, Patton MA, Nicolaides $\mathrm{KH}$. Outcome of chromosomally normal livebirths with increased fetal nuchal translucency at 10-14 weeks' gestation. $J$ Med Genet.1998;35(3):222-4.

4. Adekunle O, Gopee A, el-Sayed M, Thilaganathan B. Increased first trimester nuchal translucency: pregnancy and infant outcomes after routine screening for Down's syndrome in an unselected antenatal population. BrJ Radiol. 1999;72(857):457-60.

5. Maymon R, Jauniaux E, Cohen O, Dreazen E, Weinraub Z, Herman A. Pregnancy outcome and infant follow-up of fetuses with abnormally increased first trimester nuchal translucency. Hum Reprod. 2000;15(9):2023-7.

6. Hiippala A, Eronen M, Taipale P, Salonen R, Hiilesmaa V. Fetal nuchal translucency and normal chromosomes: a long-term follow-up study. Ultrasound Obstet Gynecol. 2001;18(1):18-22.

7. Senat MV, De Keersmaecker B, Audibert F, Montcharmont G, Frydman R, Ville Y. Pregnancy outcome in fetuses with increased nuchal translucency and normal karyotype. Prenat Diagn. 2002;22(5):345-9.

8. Cheng CC, Bahado-Singh RO, Chen SC, Tsai MS. Pregnancy outcomes with increased nuchal translucency after routine Down syndrome screening. Int J Gynaecol Obstet. 2004;84(1):5-9.

9. Senat MV, Bussieres L, Couderc S, Roume J, Rozenberg P, Bouyer J, et al. Long-term outcome of children born after a first-trimester measurement of nuchal translucency at the 99th percentile or greater with normal karyotype: a prospective study. Am J Obstet Gynecol. 2007;196(1):53 e1-6.

10. Nicolaides KH, Spencer K, Avgidou K, Faiola S, Falcon O. Multicenter study of first-trimester screening for trisomy 21 in 75821 pregnancies: results and estimation of the potential impact of individual risk-orientated two-stage first-trimester screening. Ultrasound Obstet Gynecol. 2005;25(3):221-6.

11. Pandya PP, Kondylios A, Hilbert L, Snijders RJ, Nicolaides KH. Chromosomal defects and outcome in 1015 fetuses with increased nuchal translucency. Ultrasound Obstet Gynecol.1995;5(1):15-9.

12. Snijders RJ, Noble P, Sebire N, Souka A, Nicolaides KH. UK multicentre project on assessment of risk of trisomy 21 by maternal age and fetal nuchal-translucency thickness at 10-14 weeks of gestation. Fetal Medicine Foundation First Trimester Screening Group. Lancet. 1998;352(9125):343-6.

13. Souka AP, Von Kaisenberg CS, Hyett JA, Sonek JD, Nicolaides KH. Increased nuchal translucency with normal karyotype. Am J Obstet Gynecol. 2005;192(4):1005-21.

14. Bilardo CM, Muller MA, Pajkrt E, Clur SA, van Zalen MM, Bijlsma EK. Increased nuchal translucency thickness and normal karyotype: time for parental reassurance. Ultrasound Obstet Gynecol. 2007;30(1):11-8.

15. Allen VM, Wilson RD, Cheung A, Genetics Committee of the Society of O, Gynaecologists of C, Reproductive Endocrinology Infertility Committee of the Society of O, et al. Pregnancy outcomes after assisted reproductive technology. J Obstet Gynaecol Can. 2006;28(3):220-50. 